\title{
Occupational asthma for the clinician
}

\section{Educational aims}

Occupational asthma is a "special" form of asthma that often causes diagnostic and therapeutic problems for busy clinicians. This article aims to help practising pulmonologists to:

$>1$. avoid common pitfalls that lead to the role of occupation being missed in adult patients with asthma;

$>2$. confirm the diagnosis of occupational asthma in patients with suspected occupational asthma;

$>3$. manage patients with occupational asthma.

The issues of prevention and surveillance of workers at risk of occupational asthma, however important, will not be addressed here.

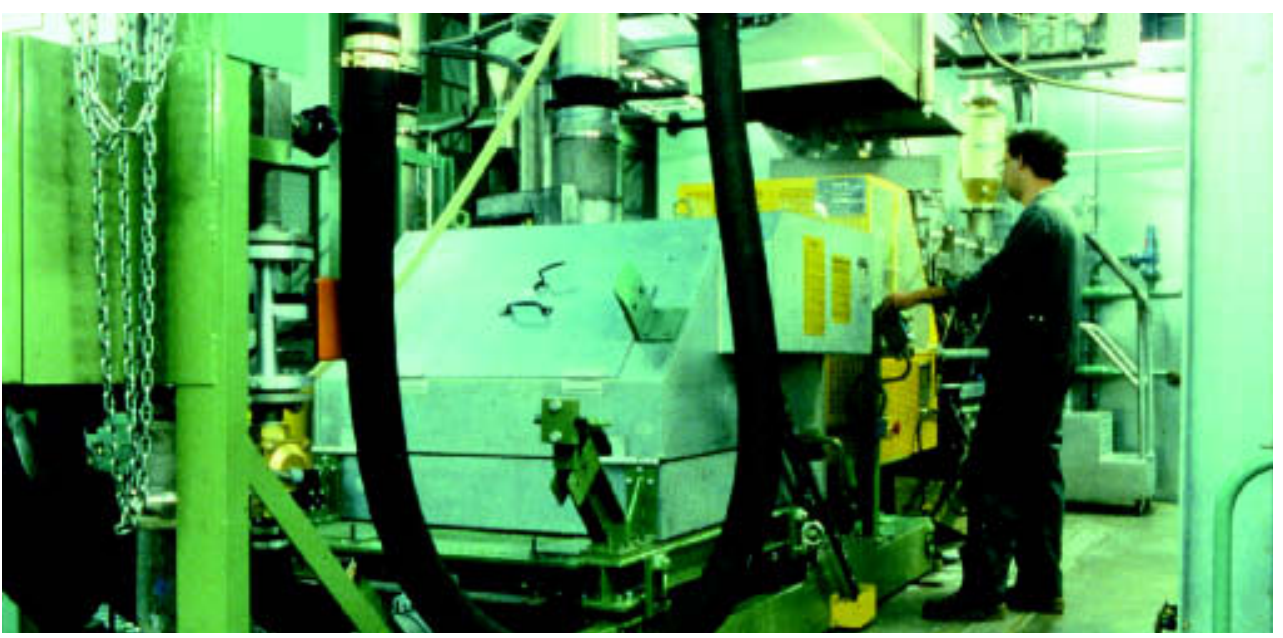

\section{Definitions}

Occupational asthma can be defined as asthma that is caused specifically by exposure to an agent present at work. The important notion in this definition is the phrase "caused specifically". This implies that when asthma is not really caused but only aggravated by work, this should not be considered as occupational asthma. Such aggravation of preexisting asthma may occur through exposure to non-specific factors, such as irritants, cold or physical exercise. However, it must be recognised that this theoretical difference between occupational asthma and work-aggravated asthma is not always easy to make in practice, particularly when there is exposure to airway irritants. Certainly, pre-existing asthma does not automatically exclude the possibility of occupational asthma, and work-aggravated asthma also needs appropriate individual and collective measures in the workplace. However, for the purposes of this article, only occupational asthma and not work-aggravated asthma will be considered.

There are different categories of occupational asthma depending on pathogenesis.

$>1$. The first category, which has also been studied most extensively, includes occupational asthma that is caused by allergic sensitisation

\section{B. Nemery}

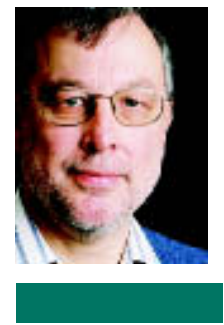

Pneumology, and Occupational, Environmental and Insurance Medicine

Katholieke Universiteit Leuven

Herestraat 49

3000 Leuven

Belgium

Fax: 3216347124

E-mail:

ben.nemery@med.kuleuven.ac.be

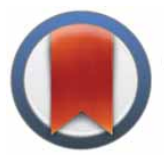

CrossMark

$\leftarrow$ click for updates

\section{Key points}

Occupational asthma is an important disease.

Its prevention is necessary, but difficult.

Its diagnosis is not always easy, but within reach of most respiratory or occupational physicians. Its correct management is complex because of the socio-economic consequences. 
Why is occupational asthma important?

\section{For the affected patient}

First of all, occupational asthma is important for the affected patient. When it can be shown that someone's asthma is caused by his/her work, this offers both therapeutic opportunities and therapeutic challenges. On the one hand, a truly causal treatment can be proposed, i.e. cessation of exposure. However, on the other hand, such treatment often brings serious "side-effects", because cessation of exposure often means job loss and corresponding socio-economic consequences. In other words, occupational asthma must not be missed so that the patient may be treated adequately, but the diagnosis must not be made too lightly either.

\section{For society}

Occupational asthma is also important for society. It has been estimated that asthma is caused directly or indirectly by work in $5-15 \%$ of adults with asthma. Even higher proportions have been recently found. Thus, in industrially developed countries, occupational asthma is the most frequent respiratory disease caused by work and it has certainly become more frequent than the traditional pneumoconioses.

\section{For science}

Occupational asthma is also important from a scientific point of view, because it has contributed to understand the pathogenesis of asthma in general. to a specific agent present in the workplace. This is the "purest" form of occupational asthma. Such occupational asthma strictu senso is also called "occupational asthma with a latency", implying that the patient has been able to do his/her work without experiencing any respiratory problems during a "latency" period of weeks to years, during which immunological sensitisation has developed. However, once sensitisation has occurred, the worker may be affected by very low concentrations of the offending agent. Such asthma generally affects only a minority of exposed workers (although the proportion of affected subjects may be substantial with potent sensitisers or poor work hygiene).

$>2$. The second category includes asthma that is not due to allergic sensitisation to a specific agent, but to exposure to irritants, such as chlorine, sulphur dioxide or acid fumes, hence the term "irritant-induced asthma". The pathogenesis of this type of asthma is much less well understood. When the causal exposure consists of a single inhalation incident, the condition is commonly called "RADS" (reactive airways dysfunction syndrome). In this instance, there is no latency between the onset of asthma symptoms and the offending exposure, hence the term "occupational asthma without latency" given to this category of occupational asthma. However, irritant-induced asthma may also be caused by repeated exposures to high levels of inhaled irritants. In such cases there is generally a period during which the worker does not yet have prominent respiratory symptoms.

$>3$. A possible third category of occupational asthma is the "asthma-like syndrome", which occurs in workers who are exposed to high levels of organic (vegetable) dusts, generally in agro-industry. The best known example of this condition is byssinosis, which occurs in cotton workers. This disease is characterised by variable airways obstruction, bronchial inflammation and evolution to chronic obstructive pulmonary disease. The main causative agent is believed to be bacterial endotoxin that originates from microbial contamination of the cotton. A similar condition has also been described in grain workers and in those engaged in intensive pig farming. Whether this condition belongs to occupational asthma or to occupational bronchitis is still unresolved.

Irritant-induced asthma and the asthma-like syndrome will not be covered further in this review.

\section{Clinical characteristics of occupational asthma Bronchial asthma Clinically, occupational asthma does} not differ from "ordinary" asthma. Typically, the patient reports episodes or attacks of breathlessness with wheezing, as well as bronchial hyperresponsiveness to non-specific triggers, including physical efforts. However, there are also presentations of occupational asthma where the patient complains mainly of chronic cough, excessive sputum production or repeated respiratory infections.

The occurrence of variable airways obstruction should be documented by spirometry with a positive bronchodilation test and/or a test of airway hyperresponsiveness with histamine or methacholine.

A differential diagnosis must be made with other pulmonary and non-pulmonary causes of episodic dyspnoea, including hyperventilation syndrome, vocal cord dysfunction, upper airway disorders, gastro-oesophageal reflux disease, etc.

\section{Relation to work}

What is typical of occupational asthma is that the symptoms are related to work. This may sound simple, but in reality the relationship to work is much more complex than is often assumed. This complexity is the main reason why the diagnosis of occupational asthma is often missed.

Sometimes the relationship between the asthma symptoms and exposures at work is quite obvious. For instance, the patient may tell spontaneously that asthma attacks occur systematically at work, e.g. soon after entering the work place or when performing specific tasks. In such instances where the patient associates his/her symptoms and work, it is important not to dismiss this possible link and to seriously consider the possibility not only of occupational asthma but also work-aggravated asthma.

However, in many instances the relationship between the asthma symptoms and 
work is not so straightforward and the patient is not necessarily aware that the symptoms are temporally related to work. This is because, even in occupational asthma, symptoms are commonly more pronounced in the evening or at night or in the early morning, i.e. outside the workplace. Another common feature of occupational asthma is that wheezing attacks may be triggered by inhaled irritants, such as cigarette smoke, engine exhaust, strong odours, fog, cold, exercise, which again occur frequently outside the workplace, e.g. in pubs or while doing sports. Asthmatics often believe that these triggers are the "cause" of their asthma ("Doctor, I am allergic to cigarette smoke").

So, a major pitfall in the clinical diagnosis of occupational asthma is that the patient with occupational asthma may have few symptoms during work and most symptoms outside the workplace. In other words, occupational asthma does not necessarily mean asthma AT work, but asthma FROM work. The practical consequence of this statement is that the clinician must not wait for patients to volunteer that their asthma may be due to work before considering the possibility of any work-relatedness.

The above considerations imply that it is generally not sufficient, nor even appropriate, to ask patients if their symptoms are worse at work. A much more efficient way to elicit a possible temporal relationship between asthma and work is to ask patients when their breathing improves. This requires a considerable reversal in the way we normally take clinical histories, because the traditional medical model focuses on conditions that cause or worsen symptoms ("What is hurting you?" "What renders you short of breath?" "What food causes you stomach trouble?"), rather than what makes the person feel better. So, in order not to overlook occupational asthma, the following question should be asked: "Does your breathing get better during week-ends or holidays?". If the patient spontaneously tells that she/he feels much better, no longer wakes up with wheezing or hardly ever needs rescue asthma medication after a few days away from work, this should strongly incite to look into triggers or real causes of asthma in the workplace. Periods away from work may consist of normal vacation, but they may also be due to sickness leave, be it for respiratory disease or not. If there are frequent periods of sickness leave for "bronchitis", then the doctor should seriously consider the possibility of these episodes being caused by work, even when these repeated bouts of bronchitis appear to be responsive to treatment with antibiotics. In such instances, it is often more likely that it is the temporary removal from exposure that is beneficial.

In addition to the frequent absence of an obvious temporal relationship between exposure and symptoms, another potential reason why the diagnosis of occupational asthma is not made timely is that occupational asthma often responds well to (adequate) medical treatment, at least initially. Unfortunately, it appears that a satisfactory control of asthma symptoms with medication often prevents both the patient and the doctor from trying to find the cause of the asthma.

Another pitfall in the diagnosis of occupational asthma is that the (good) practice of looking for allergy (by skin testing or serology) to common inhalant allergens sometimes leads to missing allergy to other agents, e.g. those present in the workplace. In other words, when an asthmatic shows allergy to house dust mite, pets or pollen, this does not automatically mean that all his/her symptoms are indeed due to the identified aeroallergens and this should certainly not exclude the possibility of occupational asthma. On the contrary, the presence of atopy is indicative of a higher risk to develop allergy to foreign proteins, including those that may be inhaled at work.

In conclusion, the clinician should always consider the possibility that asthma may have an occupational aetiology, not only in new patients, but also in well-known asthmatics.
To detect occupational asthma it is not appropriate, nor sufficient, to ask "is your asthma worse at work?"

it is more efficient to ask "does your breathing get better during the weekend or holiday?"

Burge PS. Br J Dis Chest 1987; 81: 105-115.

“Occupational asthma does not necessarily mean asthma AT work, but asthma FROM work." 


Common pitfalls that may lead to
miss a diagnosis of occupational
asthma
1. In occupational asthma, respiratory
symptoms are not necessarily most
pronounced at work, but they often
occur at home after work.
2. In occupational asthma, respiratory
symptoms are also elicited by irritants
outside work (sports, pubs, ...).
3. Occupational asthma is often
characterised by repeated absence
from work with "bronchitis".
4. Because occupational asthma
usually responds well to medication,
one is tempted not look for its cause.
5. Occupational asthma often occurs in
subjects with known allergies to
common aeroallergens (house dust
mite, pets, pollen).

"Occupational asthma may be found in very many jobs, not only in industry but also in agriculture and the service industry."

\section{Common causes of occupational asthma}

The causes of immunologically mediated occupational asthma are commonly divided into agents with high molecular weight (HMW) and agents with low molecular weight (LMW). HMW agents consist of macromolecules (generally proteins or glycoproteins) of biologic origin; LMW agents are natural or synthetic chemicals with a molecular mass $<1,500$ daltons.

\section{High molecular weight agents}

This category consists of a large variety of (glyco)proteins of animal, vegetable or microbial origin, which have the ability of causing immunoglobulin (lg)E-mediated allergic sensitisation.

$>1$. Products of animal origin may originate from mammals (farm animals, pets, laboratory animals), birds (excreta, eggs), arthropods (insects, mites), or fish and crustaceans. Allergic sensitisation and occupational asthma caused by such products can occur in people engaged in agriculture, the food industry, research, etc.

\section{$>2$. Products of plant origin comprise grain and} flour (wheat, rye), beans (soya, coffee, castor), flowers, herbs, spices, vegetables, fruit, as well as natural rubber latex (Hevea brasiliensis) or vegetable gums (acacia, guar). Occupational asthma to such plant-derived protein allergens may occur in agriculture, food, healthcare, research, transport and other sectors.

$>3$. Products of microbial origin include those derived from fungi (e.g. spores) or other microbiological agents that may contaminate food or feed, humidifiers, air-conditioning equipment or that may be encountered in biotechnology. A special category of often highly allergenic substances of microbial origin consists of enzymes (such as proteases, amylase, etc.) that are increasingly used in industrial and consumer products (detergents, food feeds, cleaning agents, pharmaceuticals, etc.).

\section{Low molecular weight agents}

It is generally accepted thatsensitisation to substances of low molecular weight results from their binding, as haptens, to proteins of the host with consequent immunological reactions. However, the exact mechanisms of these processes are still not well established and, in particular, the involvement of IgE antibodies is not always established in chemical-induced asthma.

The list of chemical agents that can cause occupational asthma is very long and ever growing with the introduction of novel chemicals in the workplace. This category of substances consists not only of synthetic chemicals, but also of metallic agents and a number of natural substances. The synthetic chemicals are often reactive chemicals that can easily bind covalently to proteins.

\section{Synthetic chemicals.}

The most prominent among the asthmogenic chemicals are the isocyanates, which have been implicated as the most common cause of chemical-induced occupational asthma. The isocyanate function consists of a $-\mathrm{N}=\mathrm{C}=\mathrm{O}$ group, which has high reactivity for alcohol $(\mathrm{OH})$ groups. This chemical property is used for making polyurethanes, which result from the condensation of diisocyanates (or polyisocyanates) with polyols. The most frequently used diisocyanates are toluene diisocyanate (TDI), methylene diphenyl diisocyanate (MDI) and hexamethylene diisocyanate (HDI). TDI and MDI are used for making polyurethane elastomers, plastics and flexible or rigid foams, which are widely utilised for insulation, seats and other vehicle parts, furniture, shoe soles, carpet backings, cable sheathing, etc. Isocyanates are also heavily used in coatings (paints, lacquers, varnish), adhesives and binders, most notably as the hardener (HDI) in two-component spray-paints for cars, but also in applications such as print-laminating and concrete flooring, or in wood products (particle/fibre board), foundry resins or sealants. Although polyurethanes have an enormous range of applications, it should be realised that not all "plastic materials" or paints are made of isocyanates.

Other chemicals that enter in the composition of polymeric materials and can cause occupational asthma are acid anhydrides, such as phthalic anhydride or trimellitic anhydride, which are used for making epoxy resins, and some acrylates, such as methyl methacrylate, 
which are used for making composites or glues. Some amines or reactive dyes are also potential causes of occupational asthma. In the health and pharmaceutical sector, occupational asthma may be caused by aldehydes, such as formaldehyde or glutaraldehyde (used for disinfection), or antiseptics, antibiotics and other drugs (and their precursors). Persulfates that are used for bleaching hair are a frequent cause of asthma in hairdressers.

Although this has not been fully established, it is reasonable to consider that chemicals that can cause allergic contact dermatitis may also cause occupational asthma if they are inhaled.

\section{Natural chemicals.}

It is not only synthetic chemicals that can cause sensitisation and asthma, but also natural chemicals. This is probably the case with occupational asthma caused by wood, such as Western red cedar, or asthma in electronic workers who are exposed to the fumes of colophony (derived from pine), which is often used as a flux for soldering in electronics.

\section{Metallic agents}

Several metallic agents can also cause sensitisation and occupational asthma. The most notorious example is asthma caused by the complex salts of platinum in refiners of precious metals. Cobalt, chromium (hexavalent chromates) and nickel are also possible causes of occupational asthma.

\section{Diagnostic approach Work-relatedness}

As indicated above, the most important thing is to take an appropriate history of the patient with asthma. Crucial elements are the occurrence of a symptom-free latency period, an improvement of symptoms when away from work, and to some extent a well-known at-risk occupation. However, the clinical history alone is generally not sufficient to make a definitive diagnosis of occupational asthma. It is essential that the causal relationship between work and symptoms be documented by objective means. Such documentation is necessary before advising a change of job and is also required if compensation is to be obtained from an official agency.
The easiest way to document the workrelatedness of asthma is to do a "stopand-resume work" test. As indicated above, the off-work period may be a normal vacation or the patient may be purposefully removed (temporarily) from normal work. The patient should then be monitored for changes in respiratory symptoms (e.g. using an asthma severity symptom score), medication need, spirometry and non-specific bronchial responsiveness. The latter must be assessed by a method allowing a good quantification of the degree of hyperresponsiveness ( $\mathrm{PC}_{20}$, i.e. provocation concentration of histamine or methacholine causing a $20 \%$ fall in forced expiratory volume in one second).

A particularly effective way of monitoring changes in pulmonary function is to ask patients to make sequential measurements of peak-flow themselves. This technique is simple and inexpensive; it has been shown to have good specificity and sensitivity; and it is nearly always feasible (unless the patient no longer works or if the patient cannot or will not cooperate). Although it is sometimes argued that the technique is subject to falsification, it can also empower the patient. The success of the procedure is heavily dependent on motivation, not only on the part of the patient, but also on the part of the physician. This implies that it is absolutely mandatory to provide adequate oral and written instructions to the patient before giving a peak-flow meter.

The sequential self-measurement of peak-flow for documenting occupational asthma requires that peak-flow be measured at least four times per day. In practice, the patient should make three forced expirations upon waking, at least twice during the day and before going to sleep. The time and the obtained values should be noted on a form (preferably not on a graph), together with indications about activities (at home or at work), symptoms and medication. To improve compliance and avoid falsifications, it is wise to specifically authorise missing values. A recording period of at least 4 weeks, with sufficient workdays and free days, is generally required to obtain interpretable results. It is essential to represent the obtained results graphically (see figs 1-3). A good method is that which depicts the daily maximal, mean and minimal values of peak expiratory
"The clinician should always consider the possibility that asthma may have an occupational aetiology, not only in new patients, but also in well-known asthmatics."

"It is mandatory to provide adequate oral and written instructions to the patient before giving a peak-flow meter." 
Figures 1-3 show examples of plots of self-measured peak expiratory flow (PEF) values in subjects with occupational asthma. Red line is the mean daily value, green and blue lines represent the daily maximum and minimum values, respectively. Coloured areas indicate days at work.

\section{Figure 1}

A graph of a 41-yr-old operative in a polyurethane factory, showing a pattern of progressive decrease in PEF over the workweek, with a partial improvement during week-ends; at the end of the recording period, the subject had to stay at home because of "bronchitis". Cause of asthma presumably a diisocyanate.

\section{Figure 2}

A graph of a 51-yr-old male operative in a car assembly plant, showing a pattern of pronounced decreases in PEF observed during workdays inside the main production hall during the first period (until day 21) no longer occurred after relocation to an outdoor job (from day 45). Cause of asthma unknown.

\section{Figure 3}

A graph of a 43-yr-old baker showing improvements in PEF on mondays offwork until day 35 and then a progressive increase during a longer period off-work. This was accompanied by a significant improvement in histamine $P C_{20}$ from 1.85 to $4.40 \mathrm{mg} \cdot \mathrm{mL}^{-1}$ (not due to a change in medication). This baker proved to be sensitised only to $\alpha$-amylase, an enzyme that is frequently added as a dough improver.

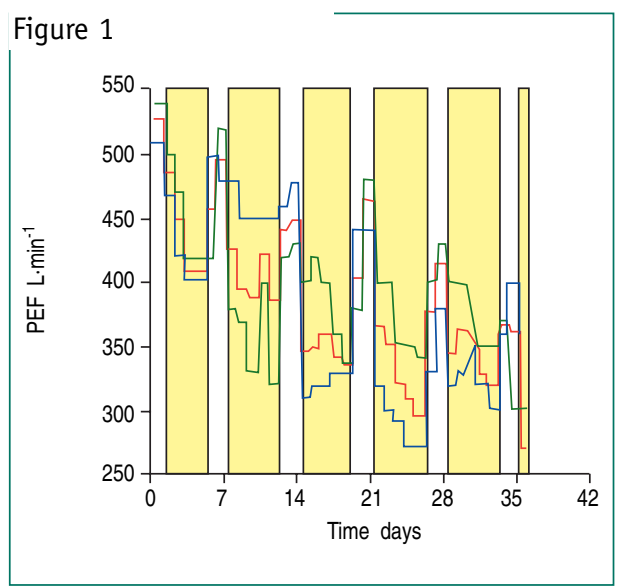

Figure 2
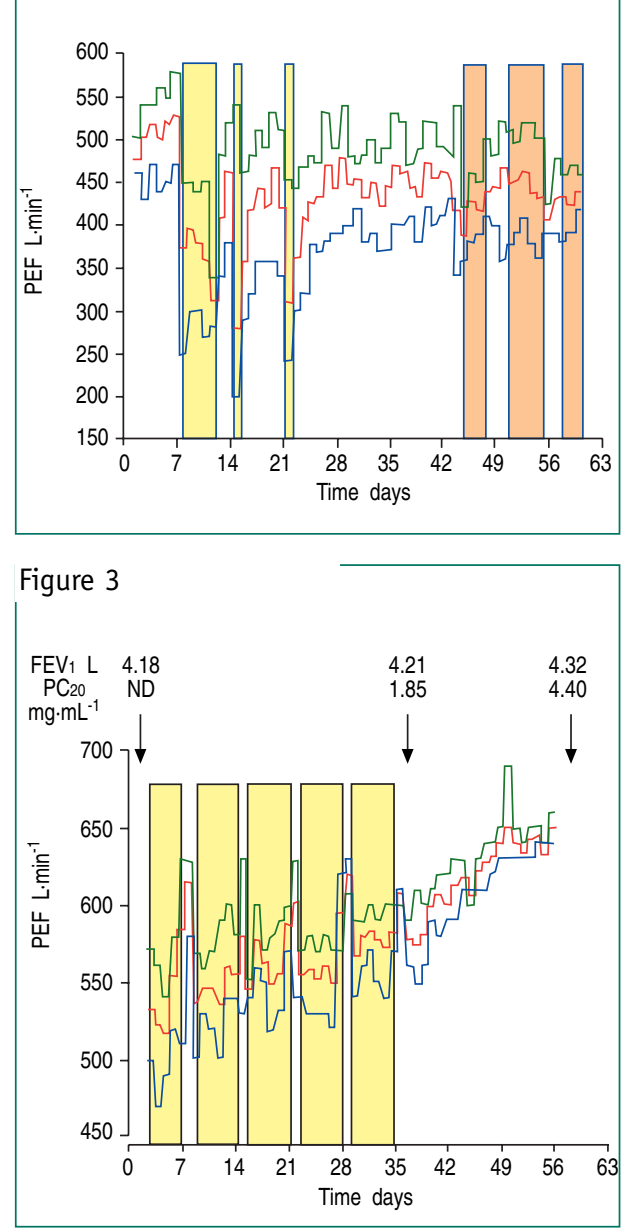

flow against time, with periods at work and off work being clearly identified. The interpretation of the results is based more on the occurrence of systematic daily or weekly patterns than on the magnitude of changes. Such interpretation may be done by "eye-balling" or by an expert-system, such as OASYS (available from P.S. Burge, Birmingham Heartlands Hospital, Birmingham,
UK). The expert system is, however, not necessarily more reliable than the subjective method.

The sequential self-measurements of PEF can be complemented by repeated measurements of the histamine or methacholine $\mathrm{PC}_{20}$. In early cases of occupational asthma, the $\mathrm{PC}_{20}$ may show appreciable changes between periods at work and periods off work. To be significant, the change in non-specific airway responsiveness must consist of at least a doubling or halving of the $\mathrm{PC}_{20}$ value, and other causes of improvement must be excluded (such as recovery from airway infection or treatment with inhaled corticosteroids). Sometimes the $\mathrm{PC}_{20}$ may normalise completely after some time without exposure.

\section{Identification of the cause of occupational asthma}

All the above procedures to try and establish the work-relatedness in occupational asthma do not allow an identification of the cause of the asthma. The causative agent can be suspected with a certain degree of confidence on the basis of the exposure circumstances. Thus, when the patient shows good evidence of having occupational asthma and is exposed to a well-known asthma-inducing agent, then it is generally reasonable to assume, for practical management purposes, that this agent is the culprit.

How can the practising physician know whether someone is exposed to an asthmainducer at work? In principle, the best source of information is the worker's occupational physician, who is ideally placed to provide data on the products used at work and their health risks, and who can also give information on other possible cases of asthma in the factory. However, this option is not always available and other methods must be tried, such as asking the worker to provide the Material Safety Data Sheets (MSDS) or labels of the products used. These documents may sometimes give useful information or at least clues as to the possible causative agents. However, one should be aware that information on the risks of respiratory sensitisation is often deficient in MSDS. Important clues to discover possible causes are recent changes in work processes, altered formulations, newly introduced ingredients or new suppliers of materials. 
In some instances it is possible to demonstrate sensitisation against a particular substance, and although this does not constitute proof that the asthma is effectively caused by this sensitiser, it is generally helpful to identify the causal agent. The demonstration of allergic sensitisation can be done with skin tests (skin-prick test or scratch test) and sometimes also with serological tests. This is generally the case for macromolecular agents, such as animal allergens, latex, flour, enzymes, etc., for which commercial extracts for skin testing or RASTs are available. If this is not the case, it is possible to dissolve the suspected material into a suitable diluent for performing skin tests. This approach is generally considered not to be applicable for low-molecular weight agents. This is particularly true for isocyanates. There is a consensus that the existing RASTs for identifying isocyanatespecific $\lg E$ antibodies are not often positive in isocyanate-induced asthma. This may be due to deficiencies in the preparation of the RASTs, but it is also possible that isocyanate-induced asthma is not necessarily mediated by $\mathrm{lgE}$ antibodies. For most low-molecular weight chemicals, there are no commercially available RASTs and skin testing is also not appropriate. However, there are exceptions to this rule, most notably in the case of asthma caused by complex platinum salts, where skin-prick testing has been shown to be both sensitive and specific for diagnosing allergy to platinum. Some reactive chemicals, such as organic dyes, can also elicit positive skin tests.

The definitive identification of the cause of occupational asthma requires a specific bronchial provocation test, which is the "gold standard" for diagnosing occupational asthma. The test is considered positive when a significant bronchospastic response is produced after the inhalation of low, i.e. non-irritant, concentrations of the suspected agent. Such responses

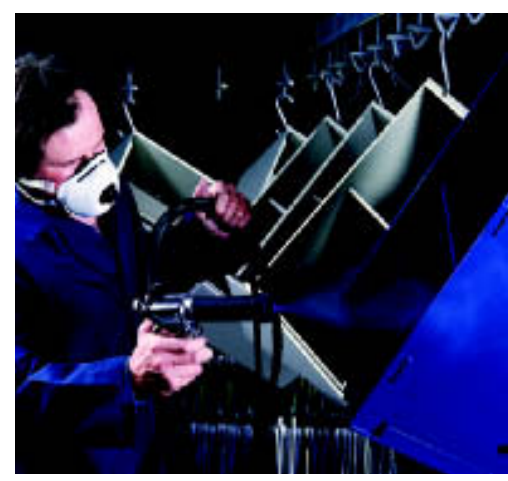

Question 10. True or false. Solvents are a frequent cause of occupational asthma in workers engaged in spraypainting, particularly in automobile spray painters.

\section{Educational questions (Answers on page 32)}

Are the following statements correct or incorrect?

1. Patients with occupational asthma may experience serious respiratory problems when practising outdoor sports.

2. The occurrence of nocturnal wheezing and cough pleads against a diagnosis of occupational asthma.

3. Inhaled corticosteroids and bronchodilators are generally effective to control respiratory symptoms in subjects with occupational asthma.

4. Occupational asthma may occur in subjects without atopy.

5. Pre-existing asthma makes a diagnosis of true occupational asthma rather unlikely.

6. Aerosolised enzymes are potent causes of occupational asthma.

7. Occupational asthma may be caused by exposure to metallic compounds, such as nickel, cobalt or chromium.

8. Latex-induced asthma in health workers is an example of asthma caused by low-molecular-weight chemicals.

9. Most plastics are made with diisocyanates.

10. See picture.

11. A recognised cause of asthma in hairdressers is the use of persulfates for hair-bleaching.

12. It is useless to make more than one measurement of nonspecific bronchial hyperresponsiveness with histamine or methacholine in patients suspected of occupational asthma.

13. Recording self-measured peak expiratory flow (PEF) over a few weeks on and off work is a generally reliable method to make a diagnosis of occupational asthma.

14. Skin-prick tests are only useful for diagnosing occupational asthma caused by sensitisation to biological agents.

15. RASTs for diisocyanates are nearly always positive in subjects with occupational asthma caused by diisocyanates.

16. Specific bronchial provocation tests should always be performed to confirm the diagnosis of occupational asthma.

17. Specific bronchial provocation challenge leads to an early or a late bronchospastic response depending on whether the causal agent is a macromolecular or a synthetic chemical agent, respectively.

18. A correct management of the patient with occupational asthma always involves complete and definitive removal from work.

19. In patients with occupational asthma, clinically significant nonspecific bronchial hyperresponsiveness may persist even after complete cessation of exposure to the causal agent.

20. Atopic subjects should be excluded from jobs with a high risk of occupational asthma, such as working with laboratory animals. 


\section{Suggested answers}

Statements 1, 3, 4, 6, 7, 11, 13 and 19 are correct.

\section{Suggested further reading}

Bernstein IL, Chan-Yeung M, Malo JL, Bernstein DI. Asthma in the workplace. 2nd ed. New York, Basel, Marcel Dekker, Inc., 1999.

This multi-author textbook is the best reference book in the field of occupational asthma. A third edition should appear in 2005.

The European Respiratory Journal has published several review articles on various aspects of occupational asthma. $A$ recent series of such articles is "Controversies in Occupational Asthma", edited by 0 . Vandenplas and J-L. Malo, of which seven articles have been published in 2003, as follows:

>1. Vandenplas O, Malo J-L. Definitions and types of work-related asthma: a nosological approach. Eur Respir $\mathrm{J}$ 2003; 21: 706-172.

>2. Moscato G, Malo JL, Bernstein D. Diagnosing occupational asthma: how, how much, how far? Eur Respir $\mathrm{J} 2003$; 21: 879-885.

> 3. Mapp CE. The role of genetic factors in occupational asthma. Eur Respir J 2003; 22: 173-178.

> 4. Sastre J, Vandenplas 0, Park HS. Pathogenesis of occupational asthma. Eur Respir J 2003; 22: 364-373. > 5. Gautrin D, Newman-Taylor AJ, Nordman H, Malo JL. Controversies in epidemiology of occupational asthma. Eur Respir J 2003; 22: 551-559.

> 6. Vandenplas 0, Toren K, Blanc PD. Health and socioeconomic impact of work-related asthma. Eur Respir J 2003; 22:689-697.

> 7. Cullinan P, Tarlo S, Nemery B. The prevention of occupational asthma. Eur Respir J 2003; 22: 853-860.

For online access to $E R J$ please go to www.erj.ersjournals.com typically occur early (within a few minutes) or late (after a few hours) or both early and late (dual response). Non-specific bronchial hyperresponsiveness may also be increased after the test (sometimes without a clear spirometric response). Specific bronchial provocation testing is not so easy to perform, it is time-consuming and costly, and it carries some risk for the patient (and the operator) and should only be done in specialised centres. Certainly such specific bronchial challenge is not always necessary to make a confident diagnosis of occupational asthma.

\section{Management of occupational asthma}

Ideally, the causal agent should be eliminated from the workplace. This option is not often available, however, and the second-best option is to remove the worker from exposure. If at all possible, such removal should be done by relocating the worker within the same company. Because even low concentrations of the allergen may cause bronchial inflammation and obstruction, the removal from exposure should be complete and definitive. Generally, it is not sufficient to let the subject work in the same hall or in the vicinity of the source of the offending agent. The installation of exhaust ventilation or wearing personal protection equipment (masks) is also often ineffective for the already affected worker, although such measures may be advised to prevent the occurrence of further cases. However, although complete cessation of exposure is desirable, it may be first worth attempting to substantially reduce the exposure (e.g. relocating a nurse with latex-induced asthma away from the operating theatre to another section in the hospital), and to evaluate the effect of this measure on symptoms, medication need, pulmonary function and non-specific bronchial hyperresponsiveness. In practice, relocation within the same company is often not possible and generally the worker must leave his/her job altogether.

Obviously, the loss of one's job has serious financial consequences for most people. This explains why the diagnosis of occupational asthma must never be made too lightly. In some countries, the medical and social consequences may be compensated by official agencies. However, it remains that for most patients with occupational asthma, the economic consequences are considerable, even when they receive compensation.

Some patients, particularly those who are self-employed, will not or cannot quit their job. In such cases, the asthma must be controlled by trying to minimise exposure, e.g. using adequate respiratory protection, and by prescribing appropriate medication. A good follow-up is necessary in these people.

\section{Prognosis of occupational asthma}

When exposure to the offending agent persists, the relation of the symptoms and pulmonary function with work may progressively become less obvious and there may be evolution towards a state of persistent asthma with a permanent deterioration of pulmonary function. Fatal asthma attacks may also occur and have been described. This must be explained to patients with occupational asthma.

Aftercessation of exposure, there is generally a gradual improvement in clinical symptoms and functional indices, including histamine tests. Such improvement may take up to 2 years. However, in a substantial proportion of patients (probably over half), there is no complete cure. Many patients remain asthmatic and keep having a certain degree of non-specific bronchial hyperresponsiveness. Some may still need anti-asthma medication. It is generally accepted that the prognosis is worse in patients who have had symptoms for a long time, i.e. when the diagnosis of occupational asthma is made late. This again demonstrates the fact that physicians must make this diagnosis as early as possible. 